\title{
Mathematical Modelling of Einstein's Rate Equations for Zinc Phosphate Glass with $\mathbf{E r}^{3+}-\mathbf{Y b}^{3+}$
}

\section{Frida Lissete Flores Rivera, Grethell Georgina Pérez-Sánchez, Miguel Angel Barron-Meza, Joan Reyes Miranda, Dulce Yolotzin Medina Velázquez*}

Division of Basic Science and Engineering, Universidad Autónoma Metropolitana, México City, México

Email: *dyolotzin@azc.uam.mx

How to cite this paper: Rivera, F.L.F., Pérez-Sánchez, G.G., Barron-Meza, M.A., Miranda, J.R. and Velázquez, D.Y.M. (2021) Mathematical Modelling of Einstein's Rate Equations for Zinc Phosphate Glass with $\mathrm{Er}^{3+}-\mathrm{Yb}^{3+}$. Open Journal of Applied Sciences, 11, 1038-1045.

https://doi.org/10.4236/ojapps.2021.119076

Received: August 23, 2021

Accepted: September 20, 2021

Published: September 23, 2021

Copyright $\odot 2021$ by author(s) and Scientific Research Publishing Inc. This work is licensed under the Creative Commons Attribution International License (CC BY 4.0).

http://creativecommons.org/licenses/by/4.0/

\begin{abstract}
There is a constant growth in the demand of data information transmission capacity, that is, more and more people send data, voice, video signals, among others, through communications networks. Due to the above there is great interest in improving network devices, such as optical amplifiers, which must cover a large bandwidth and generate greater gain than those currently available. For this reason in this work a computational simulation for a Quasi-system was carried out three energy levels of Erbium and Ytterbium varying their concentrations and proving that they are optimal candidates in a zinc phosphate matrix as this type of glass contains properties such as, high transparency, low melting point, high thermal stability, high gain density due to high solubility, low refractive index and low dispersion, which makes them optimal as signal amplifiers. The results confirm that by increasing the doping of the Erbium ion the gain of the amplifier decreases, contrary to the Ytterbium ion that by increasing the doping the gain of the amplifier increases.
\end{abstract}

\section{Keywords}

Optical Telecommunications, Rare Earths Doping, Optical Amplifier, Gain

\section{Introduction}

Amorphous solids such as phosphates have been of great importance when doped with rare earth ions, because their excellent properties have applications in solid state lasers, optical amplifiers, three-dimensional screens, among others [1].

In fact, phosphate glasses allow a high concentration of rare earth ions (RE 
rare earth) (up to 1021 ions $/ \mathrm{cm}^{3}$ ) to dissolve in the glass matrix without clumping, because of the presence of phosphorus, which introduces nonbridging oxygen (depending on its position), as shown in Figure 1, which will become a chain-like structure, compared to the random silicate glass network.

This allows the manufacture of various devices with high energy gain. In addition, this type of glass contains properties such as, high transparency, low melting point, high thermal stability, high gain density which is due to high solubility, low refractive index and low dispersion [2].

Specifically since 1995 zinc phosphate has been a case study because glass can be manufactured in a simpler way with $\mathrm{ZnO}$ contents, in addition the glass formation intervals of most other binary phosphates are generally more limited [3].

On the other hand, in optical communication networks, the information signals that travel in the optical fiber have to travel very long distances without presenting significant attenuation that prevents the recovery of the signal from the receiving side. However, when the distances covered are tens or even hundreds of kilometers, it is necessary to amplify the signal.

An optical amplifier is a device that amplifies an optical signal directly without converting it into an electrical signal. Amplified spontaneous fiber optic amplifiers provide in-line amplification of the optical input signals by the stimulated emission of photons by rare-earth ions that are implanted in the core of the fiber optic.

In recent years, the $\mathrm{Er}^{3+}-\mathrm{Yb}^{3+}$ co-doped glass waveguide (fiber optic) laser and amplifiers have been of great interest due to its advantages in cost, size, high gain, in addition to having a wide bandwidth, transparency at wavelengths and independence from polarization [4].

It is known that an Er-doped fiber optic amplifier (EDFA, Erbium-Doped Fiber Amplifier) requires a high concentration of Erbium ions $\left(\mathrm{Er}^{3+}\right)$. However, a high concentration of $\mathrm{Er}^{3+}$ will reduce the spacing between the ions, the overlap between the electron clouds becomes severe, which causes the transfer of energy between the $\mathrm{Er}^{3+}$ ions, increasing the uptake of the excited state. Clustering greatly reduces the efficiency of external excitation (pump) and degrades performance to gain.

Fortunately, the Ytterbium element, as a sensitizer, has a better overlap between the emission spectrum of the $\mathrm{Yb}^{3+}\left({ }^{2} \mathrm{~F}_{5 / 2}-{ }^{2} \mathrm{~F}_{7 / 2}\right)$ and the absorption spectrum of the $\operatorname{Er}^{3+}\left({ }^{4} \mathrm{I}_{13 / 2}{ }^{4} \mathrm{I}_{15 / 2}\right)$ also a wide and intense absorption in the wavelength range of 800 to $1080 \mathrm{~nm}$, has a weak grouping effect and a large cross-sectional absorption compared to Erbium, therefore, a high concentration of dopant $\left(\mathrm{Yb}^{3+}\right)$ can be performed in the waveguide, so the co-doping agent Erbium-Ytterbium $\left(\mathrm{Er}^{3+}-\mathrm{Yb}^{3+}\right)$ can efficiently improve the gain characteristics in the waveguide amplifiers [5] [6] [7].

On the other hand, it is important to know whether the glass is stable or tends to crystallize, as this phenomenon could take place during the manufacturing process of an optical fibre. In particular, the crystallisation temperature of the 
glass must be sufficiently above the temperature of this process to avoid the possible recrystallization phenomenon. In addition, the thermal expansion coefficient of the central glass should be slightly higher than that of the coating glass and the viscosity behaviors of the two crystals should be compatible. Reason that the viability of zinc phosphate glass as a host matrix in doping is proposed $\mathrm{Er}^{3+}-\mathrm{Yb}^{3+}[8]$

Therefore, in this project, Einstein's equations of ratio for $\mathrm{Zn}_{3}\left(\mathrm{PO}_{4}\right)_{2}$ glasses co-doped with $\mathrm{Er}^{3+}-\mathrm{Yb}^{3+}$, at different doping concentrations are mathematically modeled, confirming that they are optimal candidates as signal amplifiers.

\section{Methodology}

\section{Einstein's rate equations}

Einstein's ratio equations were implemented for a Quasi-three energy level model for the $\mathrm{Er}^{3+}-\mathrm{Yb}^{3+}$ co-doping.

In the $\mathrm{Er}^{3+} \mathrm{Yb}^{3+}$ co-doping model of Figure 1, both $\mathrm{Er}^{3+}$ and $\mathrm{Yb}^{3+}$ are excited at $980 \mathrm{~nm}, \mathrm{Yb}^{3+}$ acts as a sensitizer for the $\mathrm{Er}^{3+}$ molecules contained in the matrix. The $\mathrm{Yb}^{3+}$ ions at their excited level ${ }^{2} \mathrm{~F}_{5 / 2}$ allow the transfer of ions into the excited state of the $\mathrm{Er}^{3+}{ }^{4} \mathrm{I}_{11 / 2}$, by energy cooperation, to then have a non-radiative decay of the ions to the lower level ${ }^{4} \mathrm{I}_{13 / 2}$ of the $\mathrm{Er}^{3+}$, and finally radiatively dropping to base level ${ }^{4} \mathrm{I}_{15 / 2}$.

This gives the system two forms of pumping, through external excitation and through energy cooperation due to the sensitization of both rare earths, thus improving the emission power. The stimulated optical absorption and emission transitions are due to the pumping beam and the signal beam. For this study only the transitions between the three lowest levels are considered, assuming that the excited state to higher levels and the up conversion processes are weak. the system of equations obtained is as follows:

$$
\begin{gathered}
\frac{\mathrm{d} N_{1}}{\mathrm{~d} t}=-W_{12} N_{1}-W_{13} N_{1}+A_{21} N_{2}+W_{21} N_{2} \\
\frac{\mathrm{d} N_{2}}{\mathrm{~d} t}=W_{12} N_{1}-A_{21} N_{2}-W_{21} N_{2}+A_{32} N_{3}
\end{gathered}
$$

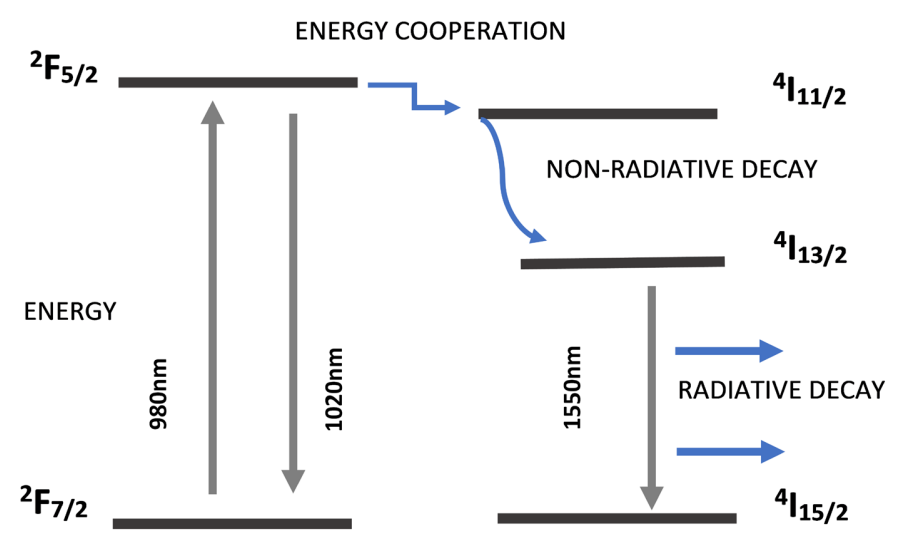

Figure 1. Energy levels and transitions in a gain medium co-doped with $\mathrm{Er}^{3+}-\mathrm{Yb}^{3+}$. 


$$
\begin{gathered}
\frac{\mathrm{d} N_{3}}{\mathrm{~d} t}=-W_{13} N_{1}-A_{32} N_{3}+A_{43} N_{4} \\
\frac{\mathrm{d} N_{4}}{\mathrm{~d} t}=-W_{45} N_{4}+A_{54} N_{5}+C_{c r} N_{1} N_{5}
\end{gathered}
$$

where:

$$
\begin{aligned}
& W(i j)=\sigma_{i j}\left(v_{S}\right) / h v_{S} \\
& W(i j)=\sigma_{i j}\left(v_{p}\right) / h v_{p}
\end{aligned}
$$

$N_{j}$ indicates the fractional population level of level $j$.

The $A_{j k}$ parameters indicate the spontaneous transition rates from level $j$ to $k$, with units of $\mathrm{s}^{-1}$.

In addition, the equations contain absorption and stimulated emission rates, which are determined by the cross-sections of each $\sigma_{j k}$ transition (whose values depend on the wavelengths involved), the optical intensities $I_{p}, I_{s}$ in the pump, the wavelength of the signal, and the photon energy $h v$.

\section{Analytical technique for solving Einstein's rate equations}

In this analytical proposal it is important to consider the following:

Assuming that $N_{1}$ and $N_{2}$ are the concentrations of $\mathrm{Er}^{3+}$ ions at levels ${ }^{4} \mathrm{I}_{15 / 2}$ and ${ }^{4} \mathrm{I}_{13 / 2}$, respectively; $N_{E r}$ is the total concentration of $\mathrm{Er}^{3+}$ ions; $N_{4}$ and $N_{5}$ are the concentrations of the $\mathrm{Yb}^{3+}$ ions at level ${ }^{2} \mathrm{~F}_{7 / 2}$ and ${ }^{2} \mathrm{~F}_{5 / 2}$, respectively; $N_{Y b}$ is the $\mathrm{Yb}^{3+}$ total ion concentration. Under the conditions of the uniform dopant and the steady state, the ion $\mathrm{Er}^{3+}$ and the ion $\mathrm{Yb}^{3+}$ at the corresponding levels depend on the wavelength guide $z$, i.e., $N_{i}=N_{i}(z)$. Therefore, the multilevel rate equations for a system co-doped with $\mathrm{Er}^{3+}-\mathrm{Yb}^{3+}$ is given by $\mathrm{Yu}$-Hai Wang et al. [5]

$$
\begin{aligned}
& \frac{\sigma_{12}\left(v_{S}\right) P_{S}(z) \Gamma_{s}}{A_{c} h v_{S}} N_{1}(z)+\frac{\sigma_{13}\left(v_{p}\right) P_{p}(z) \Gamma_{p}}{\left(A_{c} h v_{p}\right)} N_{1}(z) \\
& -\frac{\sigma_{21}\left(v_{S}\right) P_{S}(z) \Gamma_{s}}{A_{c} h v_{S}} N_{2}(z)-\frac{N_{2}(z)}{\tau_{21}}+\frac{\sigma_{45}\left(v_{p}\right) P_{p}(z) \Gamma_{p}}{A_{c} h v_{p}} N_{4}(z) \\
& -\frac{\sigma_{54}\left(v_{p}\right) P_{p}(z) \Gamma_{p}}{A_{c} h v_{p}} N_{5}(z)-\frac{N_{5}(z)}{\tau_{54}}=0
\end{aligned}
$$

With

$$
\begin{aligned}
& N_{1}(z)+N_{2}(z)=N_{E r} \\
& N_{4}(z)+N_{5}(z)=N_{Y b}
\end{aligned}
$$

where $\Gamma_{p}$ and $\Gamma_{s}$ are the overlapping factors of the pump and the signal, respectively; $A_{C}$ is the cross-sectional area of the glass; $\sigma_{12}\left(v_{S}\right)$ and $\sigma_{21}\left(v_{S}\right)$ are the cross-sectional absorption and emission signals respectively; $\sigma_{13}\left(v_{p}\right)$ the pump absorption cross-section; $\sigma_{45}\left(v_{p}\right)$ and $\sigma_{54}\left(v_{p}\right)$ are the pump absorption and emission cross-sections, respectively; $h$ is the Planck constant. Letting $P_{p}$ and $P_{s}$ be the bomb powers and steady state signal, respectively, which satisfy the following transmission equations: 


$$
\begin{gathered}
\frac{\mathrm{d} P_{p}(z)}{\mathrm{d} z}=-\Gamma_{p}\left[\sigma_{13}\left(v_{p}\right) N_{1}(z)+\sigma_{45}\left(v_{p}\right) N_{4}(z)-\sigma_{54}\left(v_{p}\right) N_{5}(z)\right] P_{p}(z) \\
\frac{\mathrm{d} P_{s}(z)}{\mathrm{d} z}=\Gamma_{s}\left[\sigma_{21}\left(v_{s}\right) N_{2}(z)-\sigma_{12}\left(v_{s}\right) N_{1}(z)\right] P_{s}(z)
\end{gathered}
$$

with

$$
\begin{gathered}
G(z)=\frac{P_{s}(z)}{P_{s}(0)} \\
\sigma=\frac{\sigma_{12}+\sigma_{21}}{\sigma_{13}+\left(\sigma_{45}+\sigma_{54}\right) \frac{1-\eta_{0}}{\eta_{0}}}\left(\sigma_{13}+\sigma_{45} \frac{N_{Y b}}{N_{E r}}\right)-\sigma_{12}
\end{gathered}
$$

where $G(z)$ will be the amplifier gain.

\section{Computer Simulator}

The mathematical model based on Einstein's rate equations was implemented in the Matlab numerical computation system.

The parameters used in the first simulation were [5].

\section{Results and Discussion}

Below are the gain graphs in $(\mathrm{dB})$ versus glass length in $(\mathrm{m})$, varying the concentration of Er by 1.0\%, 2.0\%, 3.0\%, 4.0\% and 5.0\% and leaving Yb fixed at $1.0 \%$.

And likewise varying the concentrations of $\mathrm{Yb}$ in $1.0 \%, 2.0 \%, 3.0 \%, 4.0 \%$ and $5.0 \%$, leaving Er fixed in $1.0 \%$.

In Figure 2 the values shown in Tables 1-5 are used, we can observe that in a

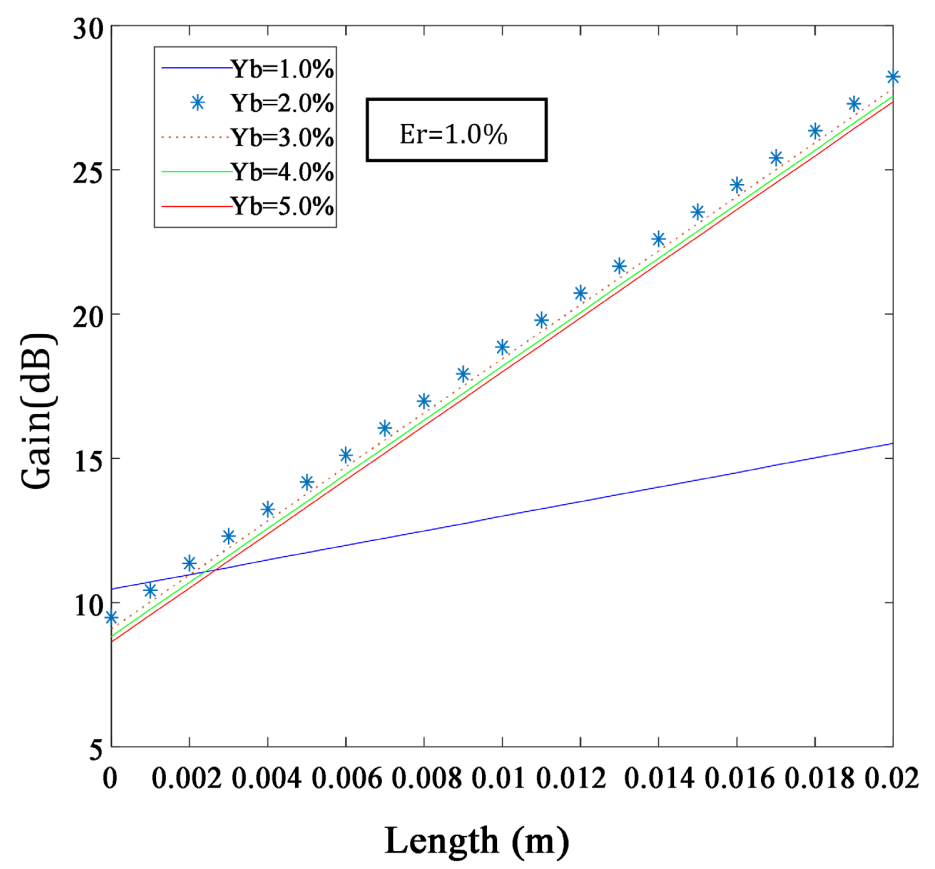

Figure 2. Representation of gain $(\mathrm{dB})$ vs Length $(\mathrm{m})$ at different concentrations of $\mathrm{Yb}$ (1.0\%, $2.0 \%, 3.0 \% 4.0 \%$ and $5.0 \%$,), Er at $1.0 \%$. 
Table 1. Parameters used for pump length and signal.

\begin{tabular}{ccc}
\hline wavelength of the pump & $\lambda_{p}$ & $980 \mathrm{~nm}$ \\
wavelength of the signal & $\lambda_{s}$ & $1550 \mathrm{~nm}$ \\
\hline
\end{tabular}

Table 2. $\mathrm{Er}^{3+}-\mathrm{Yb}^{3+}$ emission lifetime parameters.

\begin{tabular}{lcc}
\hline Emission lifetime $\mathrm{Er}^{3+}$ & $\tau_{21}$ & $10 \mathrm{~ms}$ \\
Emission lifetime $\mathrm{Yb}^{3+}$ & $\tau_{54}$ & $2 \mathrm{~ms}$ \\
\hline
\end{tabular}

Table 3. Area parameters and overlapping factors.

\begin{tabular}{ccc}
\hline Initial energy transfer & $\eta_{0}$ & $11.5 \%$ \\
Area & $A_{c}$ & $2 \times 2 \mathrm{~m}^{2}$ \\
Overlapping factor & $\Gamma_{p}$ & 0.921 \\
Overlapping factor & $\Gamma_{s}$ & 0.795 \\
\hline
\end{tabular}

Table 4. Parameters used for signal power and ion concentration $\mathrm{Er}^{3+}-\mathrm{Yb}^{3+}$.

\begin{tabular}{ccc}
\hline Signal power & $P_{s 0}$ & $1 \mu \mathrm{W}$ \\
Concentration of $\mathrm{Er}^{3+}$ ions & $N_{E r}$ & $1.0 \times 10^{26} \mathrm{~m}^{-3}$ \\
Concentration of $\mathrm{Yb}^{3+}$ ions & $N_{Y b}$ & $1.0 \times 10^{27} \mathrm{~m}^{-3}$ \\
\hline
\end{tabular}

Table 5. Parameters used in the absorption and emission cross section.

\begin{tabular}{ccc}
\hline Cross-sectional absorption of $\mathrm{Er}^{3+}$ & $\sigma_{13}\left(\lambda_{p}\right)$ & $2.58 \times 10^{-25} \mathrm{~m}^{2}$ \\
Cross-sectional absorption of $\mathrm{Yb}^{3+}$ & $\sigma_{45}\left(\lambda_{p}\right)$ & $1.0 \times 10^{-24} \mathrm{~m}^{2}$ \\
Emission cross section of $\mathrm{Yb}^{3+}$ & $\sigma_{54}\left(\lambda_{p}\right)$ & $1.0 \times 10^{-24} \mathrm{~m}^{2}$ \\
Cross-sectional absorption of $\mathrm{Er}^{3+}$ & $\sigma_{12}\left(\lambda_{s}\right)$ & $6.5 \times 10^{-25} \mathrm{~m}^{2}$ \\
Emission cross section of $\mathrm{Er}^{3+}$ & $\sigma_{21}\left(\lambda_{s}\right)$ & $9.0 \times 10^{-25} \mathrm{~m}^{2}$ \\
\hline
\end{tabular}

ratio of $\mathrm{Yb} 1.0 \%$ - Er 1.0\% you can obtain a gain of approximately $15 \mathrm{~dB}$, when now the concentration of $\mathrm{Yb}$ increases to $2.0 \%$ the gain can reach approximately $28 \mathrm{~dB}$, By further increasing the concentration of the Yterbium ion the maximum gain is maintained at approximately $28 \mathrm{~dB}$, with a length of glass up to $0.02 \mathrm{~m}$.

In Figure 3 we can observe in a ratio of $\mathrm{Er} 1.0 \%$ - Yb $1.0 \%$ which is equally 15 $\mathrm{dB}$ as in Figure 2, by increasing the concentration of the Erbium ion to $2.0 \%$ the gain decreases by about $6 \mathrm{~dB}$ and each time the concentration of Erbium is increased this phenomenon is repeated, which confirms what is established in the theoretical part where a high concentration of $\mathrm{Er}^{3+}$ will reduce the spacing between the ions, the overlap between the electron clouds becomes severe, which will cause the transfer of energy between the $\mathrm{Er}^{3+}$ ions, increasing the uptake of the excited state. Clustering greatly reduces the efficiency of external excitation (pump) and degrades performance to gain.

\section{Conclusions}

The results of the simulation confirm that by increasing the doping of the 


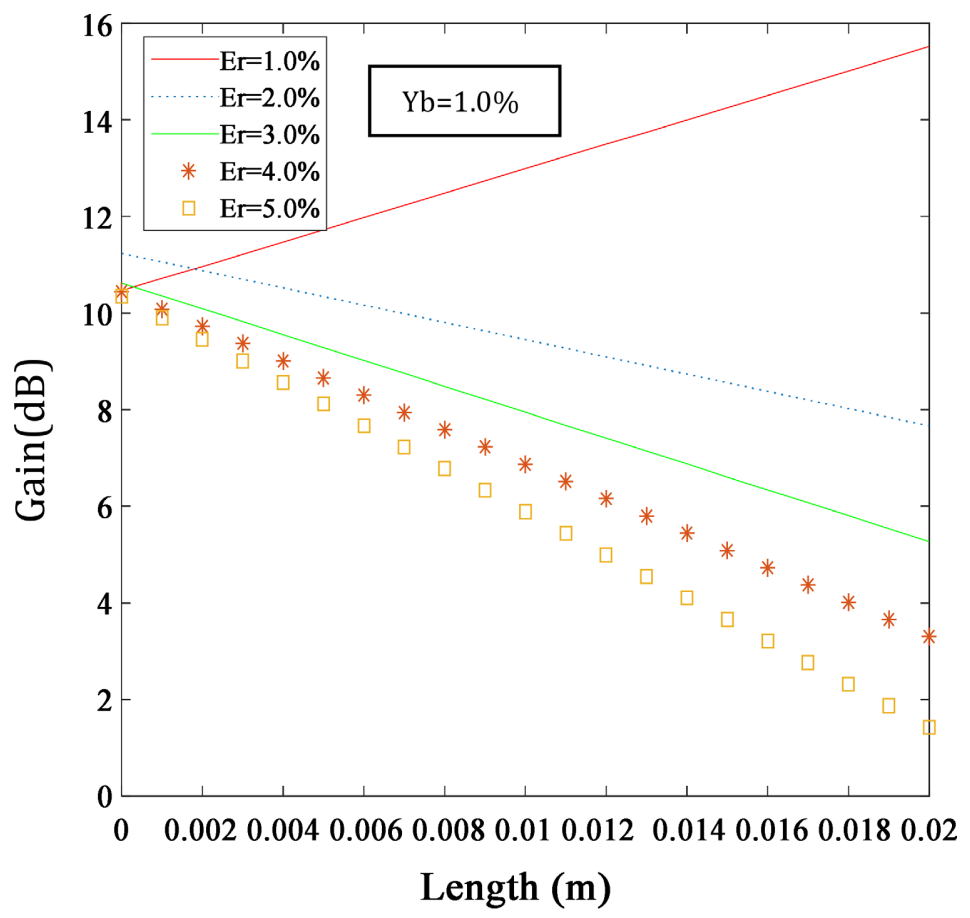

Figure 3. Representation of gain $(\mathrm{dB})$ vs Length $(\mathrm{m})$ at different concentrations of Er $(1.0 \%, 2.0 \%, 3.0 \% 4.0 \%$ and $5.0 \%)$, $\mathrm{Yb}$ at $1.0 \%$.

Erbium ion the gain of the amplifier decreases, contrary to the Ytterbium ion that by increasing the doping the gain of the amplifier increases.

With a glass length of $0.02 \mathrm{~m}$ and a concentration ratio of $1 \% \mathrm{Er}-2 \% \mathrm{Yb}$ the maximum gain obtained is $28 \mathrm{~dB}$. The highest gain was $28 \mathrm{~dB}$ with a ratio of $1 \%$ Er-2\% Yb. By increasing the concentration of Erbium by $1 \%-2 \%$ and leaving the concentration of Ytterbium fixed at $1 \%$, there is a decay of the gain of approximately $8 \mathrm{~dB}$.

From Figure 2 and Figure 3, it can be verified that the Ytterbium element, as a sensitizer, has a better overlap between the emission spectrum of $\mathrm{Yb}^{3+}$ and the absorption spectrum of $\mathrm{Er}^{3+}$, by increasing the concentration of dopant.

It will be important to consider in the future the change in amplifier gain with parameters such as pumping power in order to analyze and obtain the ideal design characteristics of the glass.

\section{Acknowledgements}

The authors are grateful for the support of CONACyT through the project through the projects 254280 and 285600, Frida Lissete Flores Rivera thanks to the National Council of Science and Technology (CONACyT-Mexico) for the scholarship received during her Postgraduate studies.

\section{Conflicts of Interest}

The authors declare no conflicts of interest regarding the publication of this paper. 


\section{References}

[1] Hraiech, S., Ferid, M., Guyot, Y. and Boulon, G. (2018) Spectroscopic Characterization and Temperature-Dependent Upconversion Behavior of $\mathrm{Er}^{3+}$ and $\mathrm{Yb}^{3+} \mathrm{Co}$-Doped Zinc Phosphate Glass. Journal of Luminescence, 197, 153-158. https://doi.org/10.1016/j.jlumin.2018.01.029

[2] Boetti, N.G., Scarpignato, G.C., Lousteau, J., Pugliese, D., Bastard, L., Broquin, J.-E. and Milanese, D. (2015) High Concentration Yb-Er Co-Doped Phosphate Glass for Optical Fiber Amplification. Journal of Optics, 17, Article ID: 065705. https://doi.org/10.1088/2040-8978/17/6/065705

[3] Brow, R.K., Tallant, D.R., Myers, S.T. and Phifer, C.C. (1995) The Short-Range Structure of Zinc Polyphosphate Glass. Journal of Non-Crystalline Solids, 191, 45-55. https://doi.org/10.1016/0022-3093(95)00289-8

[4] Vasudevan, B., Sivasubramanian, A. and Ramesh Babu, M. (2017) Optical Study on Er-Yb Co-Doped Boro-Tellurite Glasses for Optical Amplifiers. Journal of Optoelectronics and Advanced Materials, 19, 11-15.

[5] Wang, Y.-H., Ma, C.-C., Li, D.-L. and Zhang, D.-M. (2008) Formulized Analytical Technique for Gain Characteristics of Phosphate Glass $\mathrm{Er}^{3+} / \mathrm{Yb}^{3+} \mathrm{Co}$-Doped Waveguide Amplifiers. Optica Applicata, 38, 329-339.

[6] Xu, S.-H., Yang, Z.-M., Feng, Z.-M., Zhang, Q.-Y., Jiang, Z.-H., Xu, W.-C. (2009) Efficient Fibre Amplifiers Based on a Highly $\mathrm{Er}^{3+} / \mathrm{Yb}^{3+}$ Codoped Phosphate Glass-Fibre. Chinese Physics Letters, 26.

[7] Langar, A., Bouzidi, C., Elhouichet, H. and Férid, M. (2013) Er-Yb Codoped Phosphate Glasses with Improved Gain Characteristics for an Efficient $1.55 \mathrm{~mm}$ Broadband Optical Amplifiers. Journal of Luminescence, 148, 249-255. https://doi.org/10.1016/j.jlumin.2013.12.008

[8] Seneschal, K., Smektala, F., Bureau, B., Le Floch, M., Jiang, S., Luo, T., Lucas, J. and Peyghambarian, N. (2005) Properties and Structure of High Erbium Doped Phosphate Glass for Short Optical Fibers Amplifiers. Materials Research Bulletin, 40, 1433-1442. https://doi.org/10.1016/j.materresbull.2005.05.004 